\title{
GID1 expression is associated with ovule development of sexual and apomictic plants
}

\author{
Luciana Gomes Ferreira ${ }^{1,2}$ - Diva Maria de Alencar Dusi ${ }^{2}$. \\ André Southernman Teixeira Irsigler ${ }^{2}$ - Ana Cristina Meneses Mendes Gomes ${ }^{2}$. \\ Marta Adelina Mendes $^{3} \cdot$ Lucia Colombo $^{3} \cdot$ Vera Tavares de Campos Carneiro ${ }^{1,2}$
}

Received: 27 June 2017 / Accepted: 18 October 2017 / Published online: 28 October 2017

(c) Springer-Verlag GmbH Germany 2017

\begin{abstract}
Key message BbrizGID1 is expressed in the nucellus of apomictic Brachiaria brizantha, previous to aposporous initial differentiation. AtGIDIa overexpression triggers differentiation of Arabidopsis thaliana MMC-like cells, suggesting its involvement in ovule development.

Abstract GIBBERELLIN-INSENSITIVE DWARF1 (GID1) is a gibberellin receptor previously identified in plants and associated with reproductive development, including ovule formation. In this work, we characterized the Brachiaria brizantha GID1 gene (BbrizGID1). BbrizGID1 showed up to $92 \%$ similarity to GID1-like gibberellin receptors of other plants of the Poaceae family and around 58\% to GID1-like gibberellin receptors of Arabidopsis thaliana. BbrizGIDl was more expressed in ovaries at megasporogenesis than in ovaries at megagametogenesis of both sexual and apomictic plants. In ovules, BbrizGIDl transcripts were
\end{abstract}

Communicated by Xian Sheng Zhang.

Electronic supplementary material The online version of this article (doi:10.1007/s00299-017-2230-0) contains supplementary material, which is available to authorized users.

Vera Tavares de Campos Carneiro

vera.carneiro@embrapa.br

Luciana Gomes Ferreira

biolgf@yahoo.com.br

Diva Maria de Alencar Dusi

diva.dusi@embrapa.br

André Southernman Teixeira Irsigler

andre.irsigler@embrapa.br

Ana Cristina Meneses Mendes Gomes

ana.gomes@embrapa.br

Marta Adelina Mendes

martamendes86@gmail.com detected in the megaspore mother cell (MMC) of sexual and apomictic $B$. brizantha. Only in the apomictic plants, expression was also observed in the surrounding nucellar cells, a region in which aposporous initial cells differentiate to form the aposporic embryo sac. AtGIDIa ectopic expression in Arabidopsis determines the formation of MMC-like cells in the nucellus, close to the MMC, that did not own MMC identity. Our results suggest that GID1 might be involved in the proper differentiation of a single MMC during ovule development and provide valuable information on the role of GID1 in sexual and apomictic reproduction.

Keywords Apomixis - Brachiaria brizantha . Gibberellin · GID1 · Ovule development · Plant reproduction

\section{Introduction}

Gibberellins are plant hormones directly related to important plant growth and development events including seed

Lucia Colombo

lucia.colombo@unimi.it

1 Department of Biology, University of Brasília-UnB, Campus Darcy Ribeiro S/N-Asa Norte, Brasília, DF 70910-900, Brazil

2 Embrapa Genetic Resources and Biotechnology, Parque Estação Biológica, PqEB Av. W5 Norte, Caixa Postal 02372, Brasília, DF 70770-917, Brazil

3 Dipartimento di Bioscienze, Università degli Studi di Milano, Via Celoria 26, 20133 Milan, Italy 
germination, flowering and ovule development (Sun 2010; Gupta and Chakrabarty 2013; Gomez et al. 2016; Voegele et al. 2011). The gibberellin receptor GIBBERELLININSENSITIVE DWARF1 (GID1) and DELLA proteins, key negative regulators of gibberellins, act on the signal transduction pathway of gibberellin in Arabidopsis thaliana and rice (Griffiths et al. 2006; Ueguchi-Tanaka et al. 2005). The binding of gibberellin to the GID1 receptor causes a conformational change in the N-terminal extension of GID1, which inhibits gibberellin action (Sun 2010). The formation of the gibberellin-GID1-DELLA complex results in the rapid degradation of DELLA proteins, releasing the action of gibberellin by destabilizing and degrading DELLA proteins (Davière and Achard 2013; Hartweck and Olszewski 2006; Sun 2010). The GID1 gene was first identified in rice and encodes a protein with similarity to hormone-sensitive lipases (HSL) (Ueguchi-Tanaka et al. 2005). Overexpression analysis of GID1 in rice shows a hypersensitive phenotype to gibberellin, growing taller, containing long light-green leaves and with reduced fertility when compared with the control, while the gidl mutant presents insensitivity to gibberellin, suggesting that GID1 is the only gibberellin receptor in rice (Ueguchi-Tanaka et al. 2005). DELLA proteins participate in the correct formation of ovule integument and seed development in Arabidopsis, as shown in the della global mutant (the quintuple gaiT6 rgaT2 rgll-1 rgl2-1 rgl31 mutant). Short integuments and no distinction between the inner and outer cell layers of the integuments caused defects in the testa formation of della global mutant seeds. Moreover, the number of abortions increased and seed density per fruit was reduced (Gomez et al. 2016).

In angiosperms, ovule development starts with the formation of the ovule primordia from the placental tissue of the ovary. During early ovule development, a single subepidermal cell differentiates from the nucellus, leading to the formation of the megaspore mother cell (MMC). The inner and outer integuments begin to develop as the MMC undergoes meiosis, to give rise to four haploid megaspores. Three of them degenerate and the only one to survive becomes a functional megaspore, usually the one closest to the chalazal region. After three rounds of mitosis and cellularization, an embryo sac (ES) of the Polygonum-type, with three antipodal cells, a diploid central cell, two synergids, and one egg cell is formed, typical of plants of sexual reproduction (Schneitz et al. 1995; Grossniklaus and Schneitz 1998; Yang et al. 2010).

In Brachiaria, a genus of forage grass of the Poaceae family, reproduction may be sexual or asexual by apomixis, the latter resulting in progenies that are genetically identical to the mother plants (Carneiro et al. 2006). The apomictic reproduction by apospory predominates in these plants. In apospory, nucellar cells near the MMC differentiate into aposporous initial cells, which will undergo two mitosis events to form unreduced ES of the Panicum-type, with a diploid central cell, two synergids, and one egg cell. The MMC degenerates during meiosis, or complete meiosis resulting in four megaspores, which will degenerate (Araujo et al. 2000; Dusi and Willemse 1999).

In Brachiaria brizantha (Syn. Urochloa brizantha), a putative GID1-like gene of 606 nucleotides was previously detected by RNA-Seq analysis, as highly expressed in early stages of ovary development. In the model plant Arabidopsis, plant hormones were related to a fundamental role in the initiation of the ovule primordia and in the correct determination of the number of ovules (Bartrina et al. 2011; Cucinotta et al. 2014). Three genes were identified in these plants and considered as orthologous to the rice GID1, by a database search and gibberellin receptor activity of their encoded protein: AtGIDIa, AtGIDIb and AtGIDIc (Nakajima et al. 2006). Functional analysis of double mutant plants gidla gidlb showed reduced fertility; gidla gidlc also reduced fertility and showed fruits with reduced length; gidlb gidlc did not show any phenotypic difference from wild-type plants (Gallego-Giraldo et al. 2014; Griffiths et al. 2006). The triple gidla gidlb gidlc mutant showed to be extremely dwarf-sized and was insensitive to gibberellin, resulting in complete infertility. These results suggest that GID1 is the only gibberellin receptor in Arabidopsis and that this gene participates in reproductive development (Griffiths et al. 2006). The expression patterns of the three genes of Arabidopsis at anthesis, characterized using pAtGIDI::AtGIDIGUS fusion constructs, showed a GID1 a expression signal in all tissues, mainly surrounding the ES; GID1b only appeared in the chalaza region of the ovule and GID1c only in valves, with no expression in ovules (Gallego-Giraldo et al. 2014). Phenotypic traits of the gidl-1 rice mutant, such as dwarf phenotype and insensitivity to gibberellin, were restored to normal with the overexpression of AtGIDIa, AtGIDIb and AtGIDlc (Nakajima et al. 2006), showing the conserved nature of GID1 in these species. In Arabidopsis, the role of GID1 is associated with seed-set and fruit growth (Gallego-Giraldo et al. 2014); however, the role of GID1 during ovule development is still unclear. Studies related to GID1 might contribute to clarifying the mechanisms underlying the action of gibberellin in ovule development, as well as its participation in the early differential ES development of sexual and apomictic plants.

Our study aimed to evaluate the expression of GID1 during ovary development and, particularly, in the onset of ES of sexual and apomictic plants. The $B$. brizantha gene, named BbrizGID1, showed higher expression during ovule development at megasporogenesis. We detected BbrizGIDI transcripts in the MMC of sexual and apomictic B. brizantha and in the surrounding nucellar cells of the apomicts. In an attempt to mimic BbrizGIDl expression in Arabidopsis ovules, we investigated the results of overexpressing 
AtGIDla under the control of the ovule-specific SEEDSTICK promoter (pSTK) (Kooiker et al. 2005) and the 35S constitutive promoter of Cauliflower mosaic virus (p35S) (Benfey et al. 1990). Morphological characterization of reproductive organs, in particular ovules, in the transformed plants suggested that AtGIDla is involved in the differentiation of additional MMC-like cells from nucellar cells near the MMC and in the integument development, suggesting either a delayed megasporogenesis or an earlier growth of integuments in the transgenic plants. Our findings on the GID1 expression during ovule development provide valuable information on the role of GID1 in the ovule development of sexual and apomictic plants.

\section{Materials and methods}

\section{BbrizGID1 sequence analysis}

To verify the similarity of the BbrizGIDI sequence previously identified in RNA-Seq libraries of sexual and apomictic ovaries of $B$. brizantha, basic local alignment search tool (BLAST) (Altschul et al. 1990, 1997) was performed against the National Center for Biotechnology Information (NCBI; http://www.ncbi.nlm.nih.gov) database. BLASTn and BLASTx were used to compare nucleotides and translated sequences, respectively. Sequences showing the most significant nucleotide and amino acid sequence homology were used in multiple sequence alignments with ClustalW2 (Chenna et al. 2003). The cutoff $e$ value of ${ }^{>} 2 \mathrm{e}^{-57}$ and identity above $90 \%$ was used to define homologs. The Arabidopsis Information Resource database (TAIR, http://arabidopsis. org) was also used for comparative analyses between the BbrizGID1 sequence and the sequences from the Arabidopsis model plant. The genomic sequences of BbrizGIDI from sexual and apomictic genotypes were obtained searching genomic libraries under construction. Restriction map analysis of BbrizGID1 from sexual and apomictic genomes was performed with Webcutter 2.0.

\section{Plant material}

Two accessions of $B$. brizantha from Embrapa's germplasm collection, grown in the field, were used in this work: BRA 002747, a sexual diploid $(2 n=2 x=18)$, and BRA 000591, a facultative apomictic tetraploid $(2 n=4 x=36)$ named $B$. brizantha $\mathrm{cv}$. Marandu, with $98 \%$ of apospory (Araujo et al. 2007). Ovaries and anthers of both accessions were dissected from flowers of different plants before anthesis (I, II, III and IV), at different stages of development, under a Zeiss Stemi SV11 stereomicroscope. Ovaries at stages I and II contain ovules in megasporogenesis, whereas stages III and IV contain ovules in megagametogenesis (Araujo et al.
2000). The occurrence of nucellar cells and MMC characterizes ovules at stage I, in both sexual and apomictic plants. At stage II, pistils present ovules with degenerated tetrads, and in apomictic plants, aposporous initials can be visualized. At stage III, ovules with coenocytes are observed. Ovaries at stage IV contain mature ES, before anthesis (Araujo et al. 2000; Rodrigues et al. 2003). Arabidopsis wild-type (ecotype Columbia) and the pAtGIDla::AtGIDla-GUS, pAtGIDb::AtGIDIb-GUS and pAtGIDIc::AtGIDIc-GUS transgenic lines (Gallego-Giraldo et al. 2014; Suzuki et al. 2009), provided by Dr. Perez-Amador, were cultivated in a growth room at $22{ }^{\circ} \mathrm{C}$ under long-day $(16 \mathrm{~h}$ light per $8 \mathrm{~h}$ dark) conditions.

\section{Southern hybridization analysis}

Genomic DNA was extracted from young leaves of $B$. brizantha using a Dellaporta procedure (Dellaporta et al. 1983) modified by Leblanc et al. (1997). To estimate the copy number of BbrizGID1, $30 \mu \mathrm{g}$ of genomic DNA from each genotype was digested with EcoRI, HindIII and $\mathrm{XbaI}$ at $2.5 \mathrm{U} / \mu \mathrm{g}$ of DNA. A $300 \mathrm{bp}$ fragment PCR amplified with the primers GGACAGGGACTGGTACTGGAAG and CTGAGGAAGTCGGCGATCTC for BbrizGIDl without internal restriction sites for the enzymes used to digest the genomic DNA was used as probe. Labeling the probe with ${ }^{32} \mathrm{P}-\alpha \mathrm{dCTP}$, hybridization and radioactive detection assays were performed as described (Sambrook and Russell 2001). The hybridized membrane was placed in the cassette containing Imaging Plate (IP) and the image generated on the device Phosphorimager ${ }^{\text {TM }}$ FUJIFILM FLA-3000 SERIES, using the Image Reader FLA-3000 V1.8 series programs and image display by Multi Gauge V3.0.

\section{RNA isolation and RT-qPCR analyses}

In sexual and apomictic $B$. brizantha plants, total RNA was extracted in three biological replicates, each constituted of a pool of 250 ovaries, at each of the four stages, using TRIZOL $^{\circledR}$ (Invitrogen) as previously described (Rodrigues et al. 2003). Reverse transcriptase reaction was performed with SuperScript III (Invitrogen) and 2 ug of RNA according to the manufacturer's protocol. RT-qPCRs were done using SYBR Green PCR Master Mix Green (Applied Biosystems). Oligonucleotide pairs for BbrizGID1 were designed using Primer 3.0 program (Rozen and Skaletsky 2000): forward (TCAAGCTCGTGTACCGCGAG) and reverse (CTGAGG AAGTCGGCGATCTC). The analysis of data was performed by the method $\triangle \triangle \mathrm{Ct}$, with BbrizUBCE as reference gene, encoding the ubiquitin (forward GGTCTTGCTCTC CATCTGCT) and (reverse CGGGCTGTCGTCTCATAC TT), as previously used (Silveira et al. 2009). 
In Arabidopsis plants, total RNA was extracted from 1 to 2 inflorescences in three biological replicates of pSTK::AtGIDla transgenic plants and p35s::AtGIDla transgenic plants, using NucleoZOL ${ }^{\circledR}$ (Macherey-Nagel) according to the manufacturer's instructions. The total RNA was incubated with the Ambion TURBO DNA-free DNase (Invitrogen) and then reverse transcribed using the ImPromII $^{\mathrm{TM}}$ reverse transcription system (Promega). Oligonucleotide pairs were designed using Primer 3.0 program for AtGIDIa forward (GCTGCGAGCGATGAAGTT) and reverse (GAT TGGCGTTTGCAGTGA), UBIQUITIN10 forward (CTG TTCACGGAACCCAATTC) and reverse (GGAAAAAGG TCTGACCGACA), and ACTIN2-8 forward (CTCAGGTAT TGCAGACCGTATGAG) and reverse (CTGGACCTGCTT CATCATACTCTG). UBIQUITIN10 and ACTIN2-8 genes were used as references and gene expression analysis was performed using the iQ5 Multicolor real-time PCR detection system (Bio-Rad) with SYBR Green PCR Master Mix (BioRad). The assay was conducted in three technical replicates of each of the three different biological replicates for each sample.

\section{Expression analysis by in situ hybridization}

The RNA probe was synthesized using the DIG RNA labelling kit (Roche) according to the manufacturer's protocol. The same PCR fragment of 300 bp from BbrizGID1 located in the $3^{\prime}$ region, flanked by positions 1334-1633 of the nucleotide sequence, was used as a probe. The BbrizGIDI fragment was cloned into pGEM-T Easy Vector System I (Invitrogen) and used as a template for in vitro transcription with SP6 and T7 polymerases, used as sense and antisense probes, respectively. In situ hybridization was performed in semi-thin sections of $3.5 \mu \mathrm{m}$ of ovaries at megasporogenesis of sexual and apomictic plants. The sample preparation and in situ hybridization were carried out as previously described (Alves et al. 2007; Silveira et al. 2012). Hybridized sections were observed with a Zeiss Axiophot light microscope.

\section{Construction of vectors and transformation of Arabidopsis plants}

The full-length Arabidopsis AtGIDla gene was cloned using the Gateway system (Invitrogen) and the primers ATGGCT GCGAGCGATGAA and TTAACATTCCGCGTTTAC AAAC. The promoters of the SEEDSTICK gene, pSTK (Kooiker et al. 2005), to drive the expression in ovules, and the $35 \mathrm{~S}$ constitutive promoter of the Cauliflower mosaic virus (p35S) (Benfey et al. 1990) were used. Plants of Arabidopsis ecotype Columbia were transformed with p35S::AtGIDla and pSTK::AtGIDla constructs by floral dip method (Clough and Bent 1998). The pSTK::AtGIDIa transformant lines from the ecotype Columbia were selected using BASTA, and p35S::AtGIDla transformant lines from the ecotype Columbia were selected using kanamycin. The pKNU:nlsYFP Arabidopsis line (Tucker et al. 2012) provided by Dr. Tucker was also transformed with $p 35 S:$ :AtGIDla by floral dip method (Clough and Bent 1998). The transformant lines were selected using kanamycin.

\section{GUS expression analysis}

The $p$ AtGIDla::AtGIDla-GUS, pAtGIDb::AtGIDIb-GUS and $p A t G I D 1 c:: A t G I D 1 c-G U S$ lines (Gallego-Giraldo et al. 2014; Suzuki et al. 2009) were analyzed at different stages of the ovule development. $\beta$-Glucuronidase (GUS) assays were performed on inflorescences overnight at $37{ }^{\circ} \mathrm{C}$, as previously described (Liljegren et al. 2000). Samples were incubated in a clearing solution of chloral hydrate:glycerol:water (8:1:2; w/v/v), dissected and observed by DIC microscopy.

\section{Morphological analysis of Arabidopsis flowers by DIC microscopy}

Flowers at different developmental stages were collected, fixed with 9:1 ethanol:acetic acid and cleared overnight using clearing solution. After clearing, ovules were dissected from premature pistils on a slide using a stereomicroscope, mounted with a cover slip, and subsequently observed using a Zeiss Axiophot D1 microscope equipped with DIC optics. Images were captured on an Axiocam MRc5 camera (Zeiss) using the Axiovision program (version 4.1). Three different plants of wild-type (ecotype Columbia) and three independent events of each transformant line p35S::AtGIDla, pSTK::AtGIDla and the second generation of $p K N U: n l s Y F P$ p35S::AtGIDla line were analyzed. Three independent events of $p K N U: n l s Y F P$ p35S::AtGIDla line were observed with a Zeiss Axiophot microscope using a filter with $475 / 40 \mathrm{~nm}$ laser line for excitation. Emissions were detected between 530/40 nm. Images were captured on an Axiocam MRc5 camera (Zeiss) using the Axiovision program (version 4.1).

\section{Results}

\section{BbrizGID1 encodes a protein with similarity to the GID1 gibberellin receptor}

Previous RNA-Seq analysis pointed to a sequence of 606 nucleotides similar to the GID1-like gene from different plants. This sequence was present in both genomic libraries and was named BbrizGID1. Genomic libraries of sexual and apomictic $B$. brizantha (unpublished results) were screened and a full-length DNA sequence, constituted of 1646 nucleotides (Supplementary Fig. S1) and coding 352 amino acids 
(Fig. 1), was found in this analysis. The BbrizGIDl consists of two exons and one intron, similar to the GID1 structure from rice (Ueguchi-Tanaka et al. 2005). The predicted amino acid sequences of BbrizGIDl from sexual and apomictic plants have the same number of amino acids, differing in two amino acids at positions 152 and 217. In position 152, an isoleucine (I) found in sexual plants is replaced by a valine (V) in apomicts. In position 217, a lysine (K) from sexual plants is replaced by an arginine $(\mathrm{R})$ in apomicts (Supplementary Fig. S2).

To analyze the similarity of BbrizGID1 with GID1 sequences from other plants, an NCBI database search was performed. The BbrizGID1 predicted amino acid sequence showed similarity to the sequences of GID1-like gibberellin receptors of other plants of the Poaceae family (Fig. 1). The most similar sequences were from the following plants (with respective percentage identity): Setaria italica $(92 \%)$, Sorghum bicolor (91\%), Saccharum officinarum (90\%), Zea mays (87\%), Brachypodium distachyon (86\%), Oryza sativa Japonica Group (84\%) and Hordeum vulgare (83\%). The similarity of BbrizGIDl to the three Arabidopsis genes was very close: AtGIDIc (58\%), AtGIDIa (57\%) and AtGIDIb $(55 \%)$. Alignment also showed the presence of the consensus sequence of the HSL family, including the conserved HSL motifs HGG and GXSXG in BbrizGIDl (Fig. 1). The BbrizGID1 deduced amino acid sequence showed two conserved catalytic centers with the HSL sequence, serine (S) and aspartic acid (D), whereas another catalytic center, histidine $(\mathrm{H})$, was replaced by isoleucine (I) (Supplementary Fig. S3).

\section{BbrizGID1 is present in the sexual and apomictic genotypes of $B$. brizantha}

In the apomictic genotype of $B$. brizantha, the Southern blot displayed three fragments after EcoRI DNA digestion, four after HindIII and two after XbaI. In the sexual genotype, one fragment hybridized after EcoRI DNA digestion, three after HindIII and two after XbaI (Fig. 2). Restriction map analysis of genomic DNA from both genotypes (Supplementary Fig. S1) showed that there is no site for EcoRI and XbaI and a site for HindIII is found at the intron region, position 444. The probe hybridization site is between positions 1334 and 1633 from the exon region of the nucleotide sequence. Genomic hybridization confirmed that BbrizGIDl is present in both sexual and apomictic genotypes in a different restriction pattern.

\section{BbrizGID1 expression in ovaries of sexual and apomictic plants is higher during megasporogenesis}

Analysis of BbrizGIDl expression profile from the RNASeq data of ovaries of sexuals and apomicts suggested higher expression of BbrizGIDl in ovaries at megasporogenesis stage than in ovaries at megagametogenesis stage (data not shown). To confirm these results, the transcript levels of BbrizGIDl were compared quantitatively in ovaries of sexual and apomictic plants (Fig. 3), using pistils of B. brizantha in four different stages of development as previously described (Araujo et al. 2000; Rodrigues et al. 2003). Ovaries from pistils in stage $\mathrm{I}$, corresponding to the onset of megasporogenesis, showed higher expression of BbrizGID1, whereas BbrizGIDl expression decreased in the later developmental stages. At the 5\% significance level, analysis of deviance (ANODEV) test showed that sexual and apomictic reproduction modes do not interfere with BbrizGIDl expression $(F=0.903, \mathrm{GL}=19, \mathrm{GL}=1, p=0.353)$, but there are significant differences between stages I, II and III of ovule development in both plants $(F=49.66, \mathrm{GL}=19, \mathrm{GL}=3$, $p=0.0001$ ). Comparison of BbrizGIDl expression in stages III and IV, however, did not show a significant difference.

\section{BbrizGID1 is expressed in MMC of sexual and apomictic $B$. brizantha and in nucellar cells of apomicts}

In situ hybridization of BbrizGIDl was performed in the early stages of ovule development of sexual and apomictic plants to understand the cellular localization of BbrizGIDI within the initial events of ovule development (Fig. 4). The antisense probe of BbrizGIDl in the early stages of megasporogenesis resulted in high signal of hybridization in MMC of sexual (Fig. 4a, b) and apomictic plants (Fig. 4d, e). Nucellar cells in sexual ovules showed weak signal in comparison to the strong expression of BbrizGIDl in the nucellar cells of apomicts (Fig. 4d, e). In sexual and apomictic plants, a weak signal was detected in inner and outer integuments (Fig. 4a,d). The sense control probe did not show any hybridization signal in sexual and apomictic ovules, as expected (Fig. 4c, f).

\section{GID1 proteins show specific spatial expression patterns in Arabidopsis}

To study the localization of $\operatorname{GID} 1(\mathrm{a}, \mathrm{b}, \mathrm{c})$ proteins during the first stages of ovule development, transgenic Arabidopsis lines carrying $p$ AtGID1::AtGID1-GUS translational fusions (Gallego-Giraldo et al. 2014; Suzuki et al. 2009) were analyzed (Fig. 5). As visualized in the GUS assay, GID1a is present in the whole pistil during early development, before anthesis (Fig. 5a). The GUS signal was strongly detected in the inner and outer integuments of the ovules, at 2-III stage, in which MMC is in meiosis, and the inner and outer integuments already developing (Schneitz et al. 1995), of pAtGIDla::AtGIDla-GUS line. No signal was detected in MMCs and nucellar cells (Fig. 5b). The GUS signal was 


$\begin{array}{llll} & & 10 & 20\end{array}$

\begin{tabular}{l|l|l|l} 
& 90 & 100 & 110
\end{tabular}

$\begin{array}{lll} & 170 & 190\end{array}$

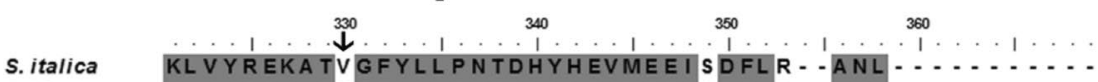
s. bicolor KLVYREKATVGFYLLPNTDHYHEVMEEIADFLR - -ANL . . . . . . s. officinarum KL VYREKA TI GFYLLSNTDHYHEVMEEI A DFLR - - ANL $\cdots \ldots .$. z. mays KVVYREKATVGFYLLSNTDHYHEVMEEI GDFLA - - ANL $\ldots \ldots \ldots$ B. distachyon KVVHREKATIGFYLLSNTDHYHEVMEEIS DFL Q - ANLL . . . . . . o. Sativa KVVQCENATVGFYLLPNTVHYHEVMEEISDFLN - ANL....... H. vulgare KVVHREKATIGFYLLSNTDHYHEVMEEIADFVQ - -LNL $\ldots \ldots \ldots$ AtGID1a KL MHLEKATVGFYLLPNNNHFHNVMDEI SAFVN - AEC . . . . . . . . AtGID1b NLLYLKQATIGFYFLPNNDHFHCLMEELNKFVHSIEDSQSKSSPVLLTP AtGID1C KLL YLEQATI GFYLLPNNNHFHTVMDEIAAFVN - AECQ........ BbrizGID1 KLVYREKATI GFYLLPNTDHYHEVMEEIADFLR - ANL.........

Fig. 1 Multiple sequence alignment by ClustalW of BbrizGID1 sequence, from the apomictic plants, showing the Arabidopsis thaliana and the most similar sequences found: Setaria italica (Accession Number XP_004962116.1), Sorghum bicolor (XP_021303311.1), Saccharum officinarum (CAP64326.1), Zea mays (CAP64327.1), Brachypodium distachyon (XP_003568469.1), Oryza sativa japonica group (XP_015639961.1), Hordeum vulgare (CAO98733.1), AtGIDla (At3g05120), AtGIDlb (At3g63010) and AtGID1c (At5g27320). Gray highlight shows the amino acid residue conserved amongst the sequences. Squares represent regions identified as conserved within the hormone-sensitive lipase (HSL) family, HGG and GXSXG motifs. Black arrows show the three catalytic centers, serine (S), aspartic acid (D), and isoleucine (I), which replace histidine $(\mathrm{H})$, characteristic of the HSL family in all the GID1 sequences of the Poaceae plants. Numbers indicate the position from the start codon. White arrows indicate glycine $(\mathrm{G})$ and arginine $(\mathrm{R})$ residues that are essentials for maintaining the gibberellin binding activity in OsGID1 in rice (Ueguchi-Tanaka et al. 2005) 
Fig. 2 Genomic DNA analysis of apomictic (APO) and sexual (SEX) Brachiaria brizantha digested with EcoRI (E);

Hind III (H) and XbaI (X). a Electrophoresis in $0.8 \%$ agarose gel in b fragments hybridized in Southern blot analysis with a BbrizGID $1{ }^{32} \mathrm{P}$-labeled probe. In APO three fragments were displayed after EcoRI DNA digestion, four after HindIII digestion and two after $\mathrm{XbaI}$ digestion. In SEX, one fragment was hybridized after EcoRI digestion, three after HindIII and two fragments after $\mathrm{Xba \textrm {I }}$ digestion. Image was generated on a phosphor imaging plate. $\mathrm{C}$ as control, the same fragment used to synthesize the probe

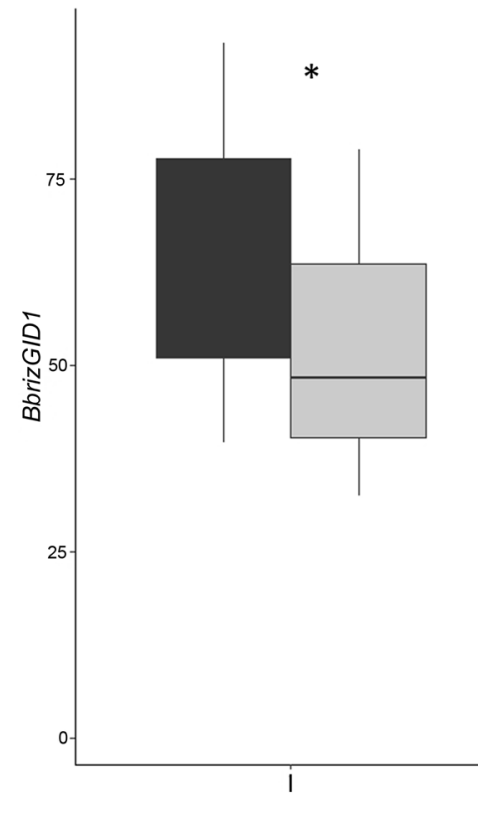

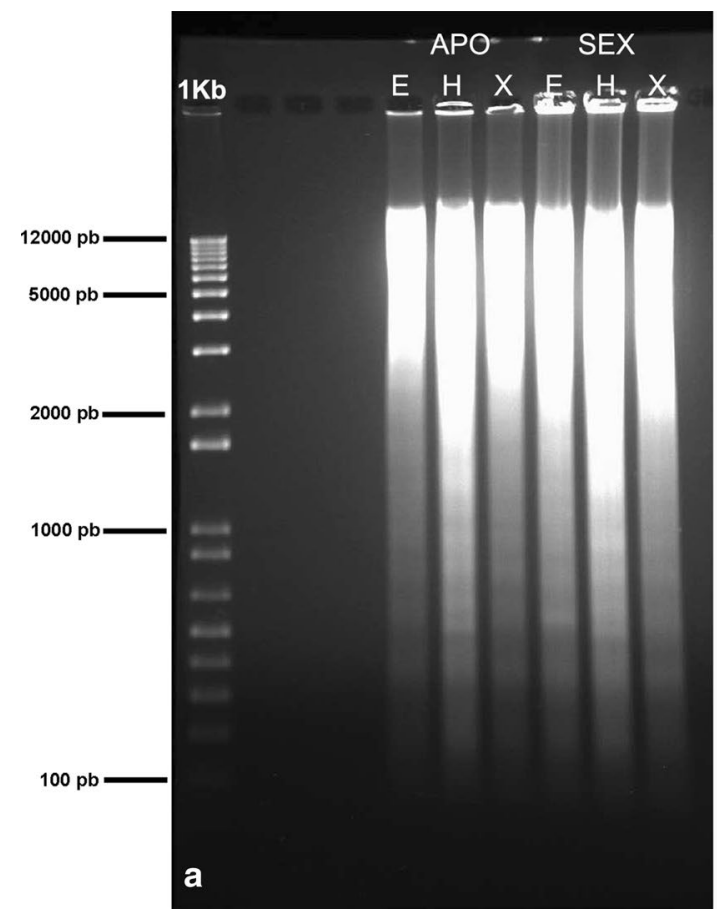

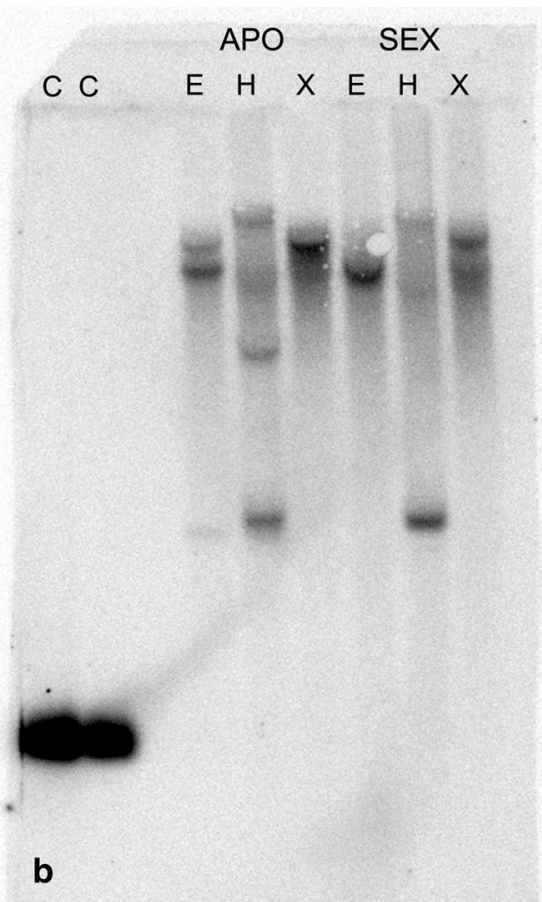

b
Fig. 3 Boxplot showing the relative expression levels of the BbrizGID1 by RT-qPCR in Brachiaria brizantha. Analysis in developing ovaries before anthesis at megasporogenesis (stages I, II) and megagametogenesis (stages III and IV) of apomictic (APO) and sexual (SEX) plants. Bars represent standard deviation based on the

present throughout the mature ovule, including the ES and the integuments (Fig. 5c). In the pAtGIDIb::AtGIDIb$G U S$ line GUS signal was localized in the inner and outer integuments at 2-III-2-V stage (Schneitz et al. 1995) and no mean of three biological samples. The mean quartiles, median and ANODEV were calculated in the $\mathrm{R}$ Core Team (2016) program. The significant differences between stages of ovary development are marked with asterisks $(* p<0.05)$

signal was detected in MMCs and nucellar cells (Fig. 5d, e). In mature ovules, at the FG7 stage in which the ES is formed, the GUS signal was localized mainly in the chalaza zone, at the base of the ES (Fig. 5f). AtGIDIc was expressed 


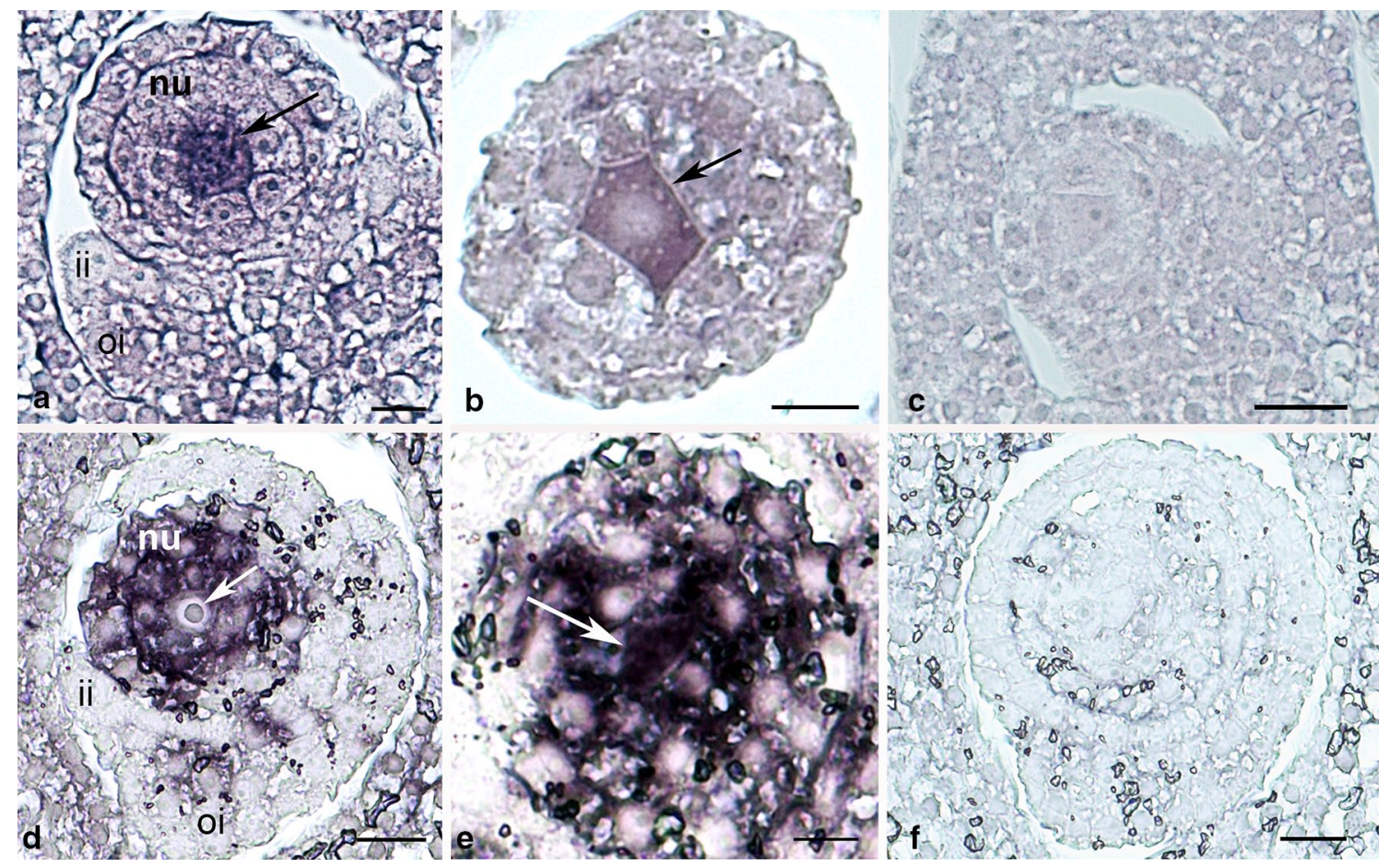

Fig. 4 Localization of BbrizGID1 expression by in situ hybridization in semi-thin sections of ovaries of Brachiaria brizantha sexual $(\mathbf{a}-\mathbf{c})$ and apomictic (d-f) plants. a Ovule with labeling in the megaspore mother cell (arrow) with weaker signal in the nucellar cells (nu), inner (ii) and outer (oi) integuments. b Detail of the megaspore mother cell (arrow) with strong signal of hybridization. c Control

specifically in the septum, but was completely absent in the ovules at 2-III stage and funiculi of pAtGID1c::AtGIDIcGUS line (Fig. 5g, h). GUS expression was not observed in ovules.

\section{Phenotypic characteristics of Arabidopsis overexpressed AtGID1a lines are distinct from wild-type}

To study whether ectopic AtGIDla was sufficient to determine the formation of MMC-like cell we have transformed Arabidopsis plants with p35S::AtGIDla and $p S T K:: A t G I D l a$. pSTK is specifically active during ovule development starting from stage I (Brambilla et al. 2008). We analyzed three transgenic plants containing p35S::AtGIDla and three containing $p S T K:: A t G I D 1 a$ by RT-qPCR. Results showed that AtGIDla expression was higher in the inflorescences of plants from both groups of transgenic lines compared to wild-type plants (Supplementary Fig. S4). The early stages of ovule development of these transgenics were analyzed, especially the finger-like (2-I) stage, in which MMC is formed (Schneitz et al. 1995) and additional MMC-like cells were observed (Fig. 6). The $p S T K: \because A t G I D 1 a$ and $p 35 S:: A t G I D 1 a$ plants showed asynchrony in development of the inner and outer with sense probe showing no signal. d Detail of the ovule with labeling in the nucellar cells (nu) and the megaspore mother cell (arrow) with weaker signal in the inner (ii) and outer (oi) integuments. e Next section of the anterior ovule showing the cytoplasm of the megaspore mother cell (arrow). f Control with sense probe showing no signal. Scale bars a $12 \mu \mathrm{m}, \mathbf{b} 10 \mu \mathrm{m}, \mathbf{c} 20 \mu \mathrm{m}$ and $\mathbf{d}-\mathbf{f} 10 \mu \mathrm{m}$

integuments as regards megasporogenesis events if compared with wild-type (Fig. 6). These results suggest that ectopic expression of AtGIDla could interfere with proper integument development. The additional MMC-like cells of overexpressed AtGIDla plants were observed only in ovules at the finger-like stage (2-I). In later stages of development, only one functional megaspore was observed, suggesting that only one MMC completed the meiotic division and entered the gametogenesis process, as twin ES were not observed during the analysis of mature ovules. Results of the phenotype analysis of wild-type Arabidopsis and three lines of $p S T K:: A t G I D 1 a, p 35 S:: A t G I D 1 a$ were evaluated for each plant (Supplementary Table S1). In ovules of wild-type Arabidopsis, additional MMC-like cells were also observed but in a reduced number, $17 \%$ of the total number of ovules analyzed. In transgenic plants, additional MMC-like cells were observed in 33 and $32 \%$ of the total number of ovules analyzed using pSTK or p35S promoter, respectively (Fig. 7). Statistical analysis (t test) showed that there is a significant difference between the number of additional MMC-like cells in the overexpressed AtGIDIa lines compared to wild-type, independent of the promoter used for AtGIDla expression, pSTK $(p=0.009)$ or $\mathrm{p} 35 \mathrm{~S}(p=0.013)$. In $p S T K:: A t G I D 1 a$ and 

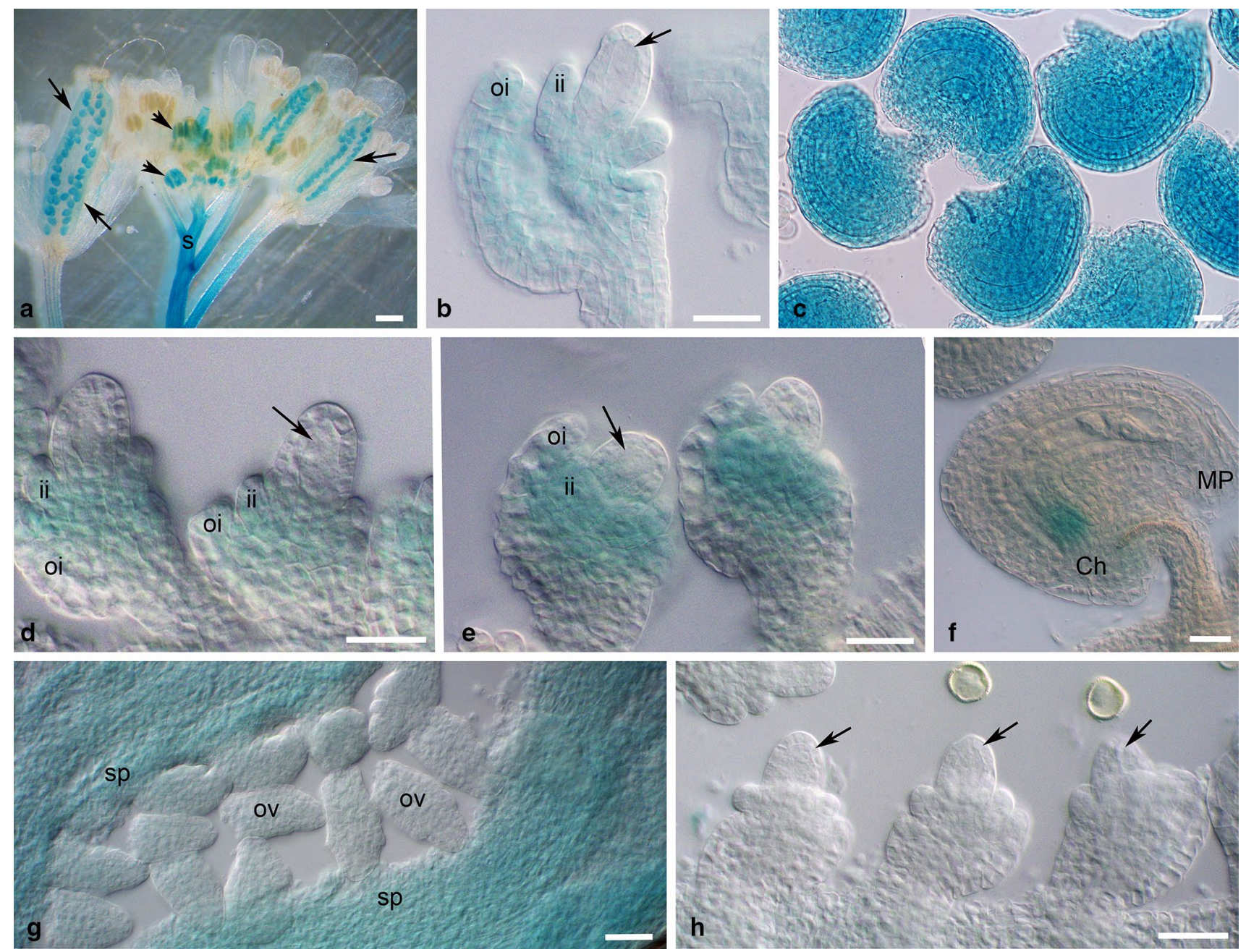

Fig. 5 Histochemical GUS analysis in inflorescences and ovules of Arabidopsis thaliana pAtGID1::AtGID1-GUS transgenic lines before anthesis. a-c pAtGIDla::AtGIDla-GUS line; d-f pAtGIDIb::AtGIDIb-GUS line and $\mathbf{g}, \mathbf{h}$ pAtGID1c::AtGID1c-GUS line. a GUS staining can be observed in stem (S), ovules (arrows) and anthers (arrow heads). b At 2-III stage of the ovule development GUS signal was localized in the inner (ii) and the outer (oi) integuments, the expression was not detected in MMC in meiosis (arrow) and nucellar cells. $\mathbf{c}$ In the mature ovules the GUS signal was localized throughout the embryo sac and the integuments. d In the 2-III stage

p35S::AtGIDla plants the number of seeds per silique increased relatively to wild-type. The transgenic plants from both genotypes showed an average of 55 seeds per silique in the 30 siliques counted with $1.5 \%$ of aborted seeds, while wild-type plants, under the same environmental conditions, presented an average of 46 seeds per silique in the 32 siliques counted with $2.5 \%$ of abortion rate. Statistical analysis ( $t$ test) showed a significant difference between the number of seeds of the transgenic plants ectopically expressing AtGIDla, independent of the promoter, $\operatorname{pSTK}(p=0.0009)$ or $\mathrm{p} 35 \mathrm{~S}(p=0.001)$ in respect of the wild-type plants (Fig. 8, Supplementary Table S1). and e first stage of gametogenesis, GUS signal was localized in the inner (ii) and outer (oi) integuments of the ovules, the expression was not detected in MMC in meiosis (arrow) and nucellar cells. $f$ In the mature embryo sac the GUS signal was localized mainly in the chalaza (Ch) in comparison with the micropyle region (MP). g GUS signal was localized specifically in the septum (sp), but was completely absent in the ovules (ov) and funiculi. h GUS signal was undetectable in ovules, arrows showing the MMC in meiosis and the nucellar cells. $M M C$ megaspore mother cell; Scale bars a $1 \mathrm{~mm}$; b-f $20 \mu \mathrm{m}$ and $\mathbf{g}$, h $50 \mu \mathrm{m}$

To investigate if the additional MMC-like cells had MMC identity, a $p K N U: n l s Y F P$ line (Tucker et al. 2012) was transformed with $p 35 S:: A t G I D 1 a$. The second generation of transgenic lines overexpressing AtGIDla showing $28 \%$ of additional MMC-like cells was analyzed (Supplementary Table S1). Plants $p K N U: n l s Y F P$ p35S::AtGID1a showed only one cell expressing the YFP signal, indicating that one out of the two MMC-like cells acquired the proper MMC identity (Fig. 9). 

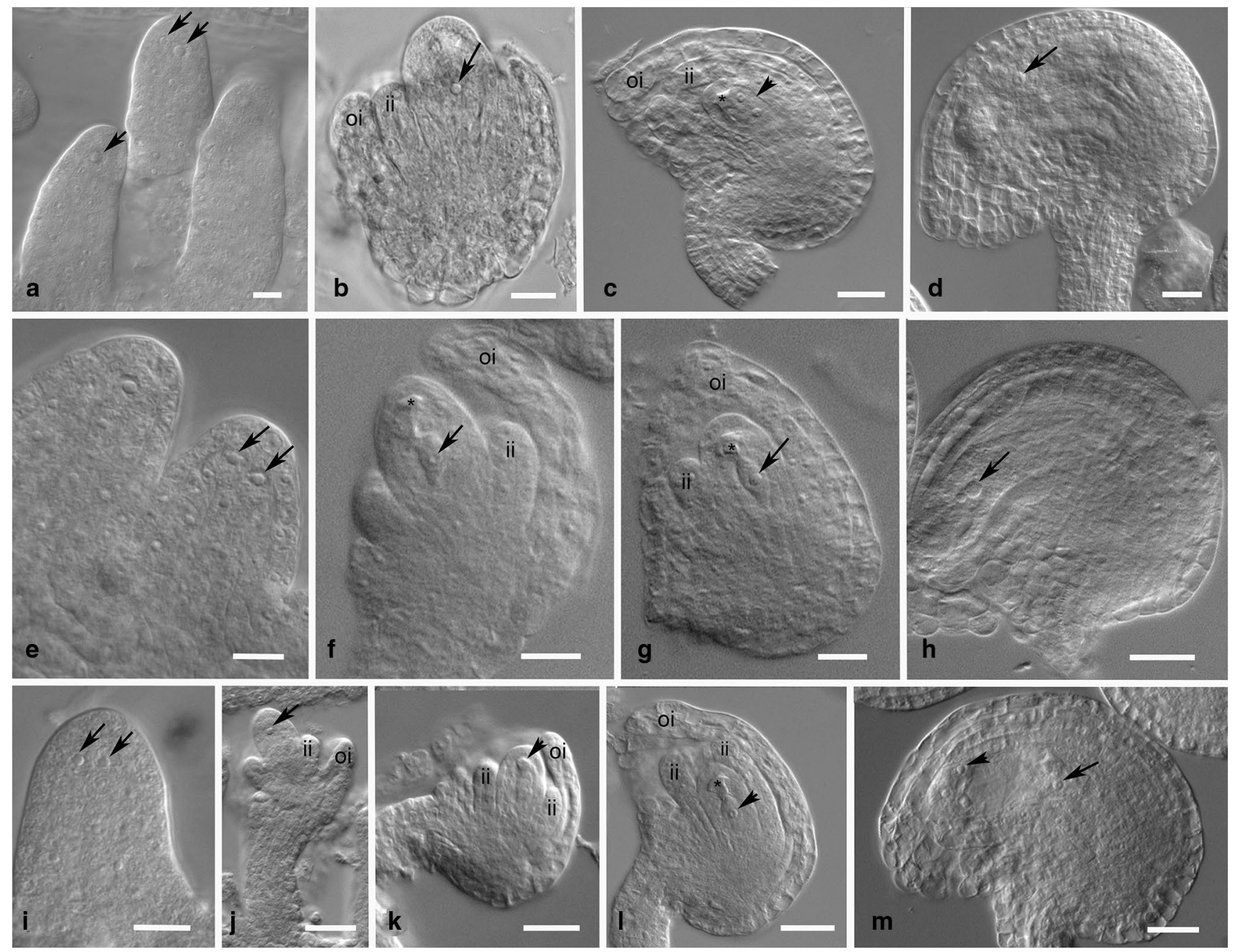

Fig. 6 Ovules of wild-type and AtGIDla overexpression lines of Arabidopsis thaliana. a-d Wild-type showing normal female gametophyte development. a Finger-like stage (2-I) showing MMC-like cells (arrows). b FG1 stage displaying MMC (arrow) integument development. c FG3 with degenerated megaspores and FG. d FG6 stage, arrow indicates the central cell. e-h p35S::AtGIDla line. e Fingerlike stage (2-I), showing MMC-like cells (arrows). f, g FG1 stage showing well-developed integuments. h FG6 stage, arrow indicates the central cell. i-n $p S T K:: A t G I D 1 a$ line. i Finger-like stage (2-I), viewing MMC-like cells (arrows). j showing MMC (arrow) and inner

\section{Discussion}

GID1 acts in the activation of gibberellin via degradation of DELLA proteins (Davière and Achard 2013; Ueguchi-Tanaka et al. 2007). The detection of GID1 in the transcriptome database of ovaries of sexual and apomictic $B$. brizantha led us to investigate a putative role of this gene in the differential ovule development of sexual and apomictic plants. Alignment of the BbrizGIDl sequence in databases showed high similarity with sequences from other species of the Poaceae family and the conserved motifs, HGG and GXSXG, from the HSL family. It was suggested that three conserved amino

(ii) and outer (oi) integuments already growing. k Tetrad (arrowhead) and growing inner (ii) and outer (oi) integuments. I FG1 stage showing functional megaspore (arrowhead), degenerated megaspores and well-developed integuments $\mathbf{m}$ FG6 stage with antipodal cells (arrow) and three nuclei at the micropylar pole (arrowhead). ii Inner integuments, oi outer integuments, $M M C$ megaspore mother cell, asterisk degenerated megaspores, $F G$ female gametophyte. Scale bars a $20 \mu \mathrm{m} ; \mathbf{b ~} 10 \mu \mathrm{m} ; \mathbf{c} 20 \mu \mathrm{m} ; \mathbf{d} 40 \mu \mathrm{m} ; \mathbf{e} 20 \mu \mathrm{m} ; \mathbf{f}, \mathbf{g} 50 \mu \mathrm{m} ; \mathbf{h} 100 \mu \mathrm{m} ; \mathbf{i}$ $50 \mu \mathrm{m} ; \mathbf{j}-\mathbf{l} 10 \mu \mathrm{m}$ and $\mathbf{m} 20 \mu \mathrm{m}$

acids of the HSL family form the catalytic triad center: serine (S), aspartic acid (D) and histidine (H) (Osterlund 2001). In all the GIDI sequences of the Poaceae plants, in which similarity to BbrizGIDl was detected, replacements of residues from this HSL catalytic center were verified. In rice, a replacement of the $\mathrm{H}$ by valine $(\mathrm{V})$ did not affect the binding activity with gibberellin, although an absence of enzymatic activity was detected (Ueguchi-Tanaka et al. 2005). In $B$. brizantha, this same $\mathrm{H}$ of the catalytic triad is replaced by isoleucine (I) but $\mathrm{S}$ and $\mathrm{D}$ are conserved. The residues glicina $(\mathrm{G})$ e arginina $(\mathrm{R})$ are essential to maintain binding between the receptor and the bioactive gibberellin in rice 
Fig. 7 Comparison among the number of ovules with additional MMC-like cells of wild-type and transgenic lines $p S T K:: A t G I D l a$ and p35S::AtGID1a of Arabidopsis thaliana. Different letters indicate statistically significant differences between the number of additional MMC-like cells of transgenic plants and wild-type, independent of the promoter used for AtGIDla overexpression, $\operatorname{pSTK}(\mathrm{p}=0.009)$ or $\mathrm{p} 35 \mathrm{~S}$ $(p=0.013) . W T$ wild-type, $N$ number of ovules analyzed, $M M C$ megaspore mother cell

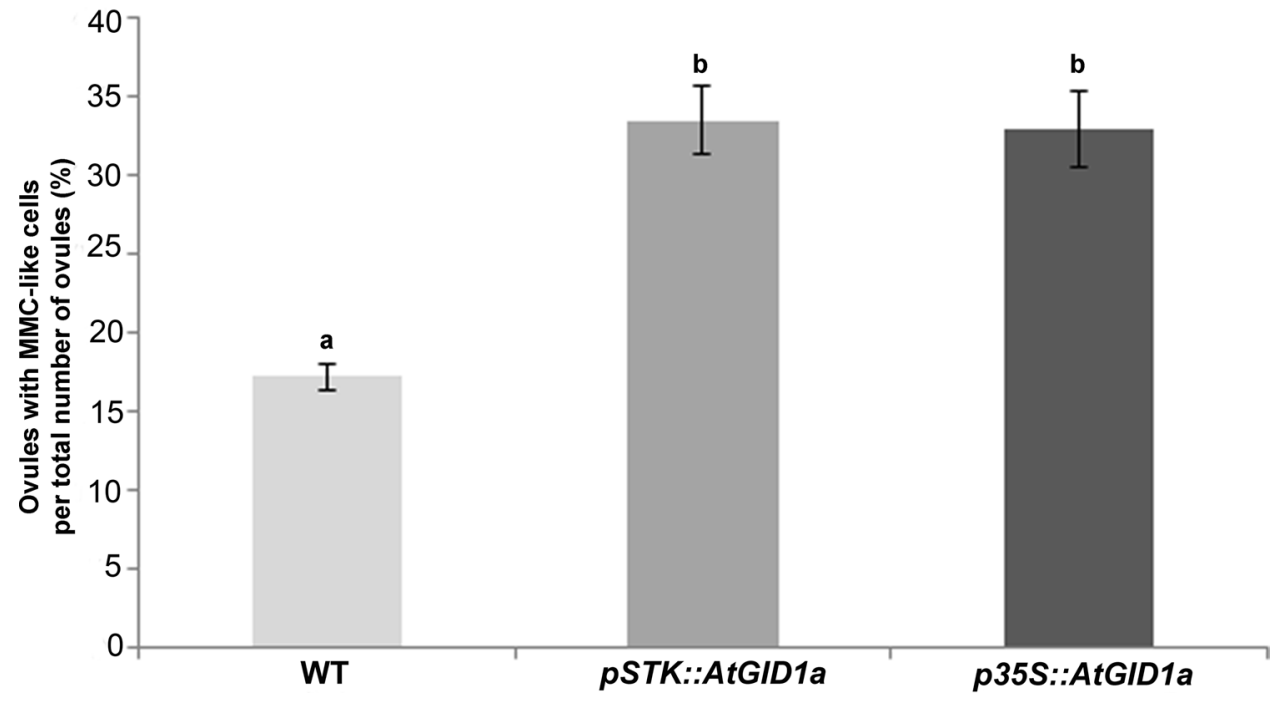

(Nakajima et al. 2006; Ueguchi-Tanaka et al. 2005). Comparing the GIDl sequence of B. brizantha with Arabidopsis revealed around $60 \%$ similarity of the BbrizGID1 sequence with the three genes AtGIDIa, AtGIDIb and AtGIDIc. In Arabidopsis, analysis of mutant combinations of the three genes showed an overlap of function among them (Griffiths et al. 2006). Complementation with each AtGID1 gene rescued the dwarf phenotype of the rice gibberellin-insensitive mutant, showing conservation of function among them (Nakajima et al. 2006). BbrizGIDl is present in the sexual and apomictic genotypes of $B$. brizantha. The restriction pattern of DNA from these plants, observed after HindIII digestion and genomic hybridization with BbrizGIDl probe, suggested a possible different number of copies of the gene in sexual and apomictic plants.

Quantitative analysis of BbrizGIDl expression showed the highest expression level in ovaries at early

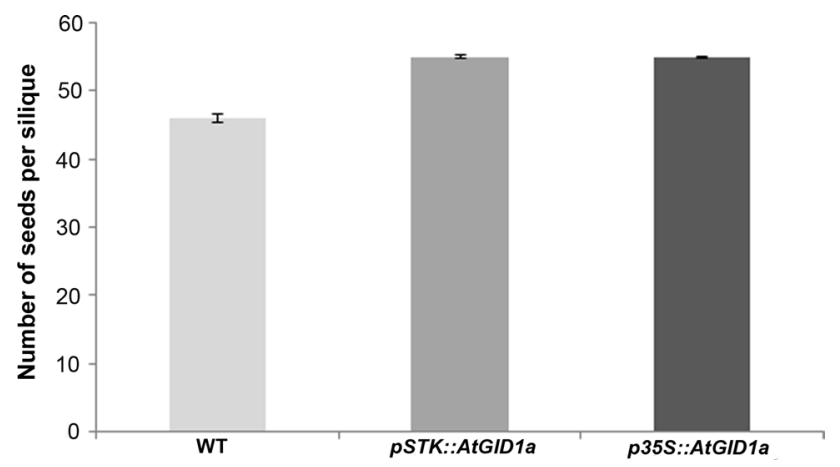

Fig. 8 Comparison among the number of seeds per silique in pSTK::AtGIDla and p35S::AtGID1a Arabidopsis thaliana relatively to wild-type (WT) plants. Different letters indicate statistically significant differences between the number of seeds of the wild-type and the transgenic plants ectopically expressing AtGIDla, independent of the promoter used, pSTK $(p=0.0009)$ or $\mathrm{p} 35 \mathrm{~S}(p=0.001)$ megasporogenesis, decreasing in the subsequent stages, independent of the reproductive mode of the plants. At this stage the formation of the MMC in sexual and apomictic genotypes was previously observed, but aposporous initial cells were still not detected in apomicts (Araujo et al. 2000). Analysis of these results suggested that BbrizGIDl might participate in the early development of ovaries of sexual and apomictic $B$. brizantha, when growing integuments and the differentiation of MMC from nucellar cells occur. Localization of transcripts of BbrizGIDl in ovules of B. brizantha from both reproductive modes revealed that MMC is expressing this gene, while in the surrounding nucellar cells the expression occurs only in the apomicts. These results suggested association of BbrizGIDl expression not only with the development of MMC but also with the nucellar cells, previous to the differentiation of aposporous initials.

The overexpression of the AtGIDla gene in Arabidopsis significantly increased the number of additional MMC-like cells of the wild-type Columbia ecotype, suggesting that this gene might be associated with the differentiation of an additional MMC-like cell in the nucellus. Analysis of the second generation of $p K N U: n l s Y F P$ p35S::AtGIDla suggested that the trait was stably inherited. Different ratios of additional MMC-like cells were previously reported in Arabidopsis ecotypes (Rodríguez-Leal et al. 2015). Analysis of ago 9 mutants showed a percentage of abnormally MMC-like cells varying from 37.16 to $47.7 \%$, with identity to gametic cells that do not undergo meiosis, a phenotype that resembles apomictic reproduction by apospory (Olmedo-Monfil et al. 2010). In our work, the identity of MMC was investigated by the expression of YFP marker genes under the control of the KNUCKLES promoter, which drives expression to the MMC (Tucker et al. 2012). Arabidopsis pKNU:nlsYFP line, overexpressing AtGIDla, showed only one cell with MMC identity in each ovule, 


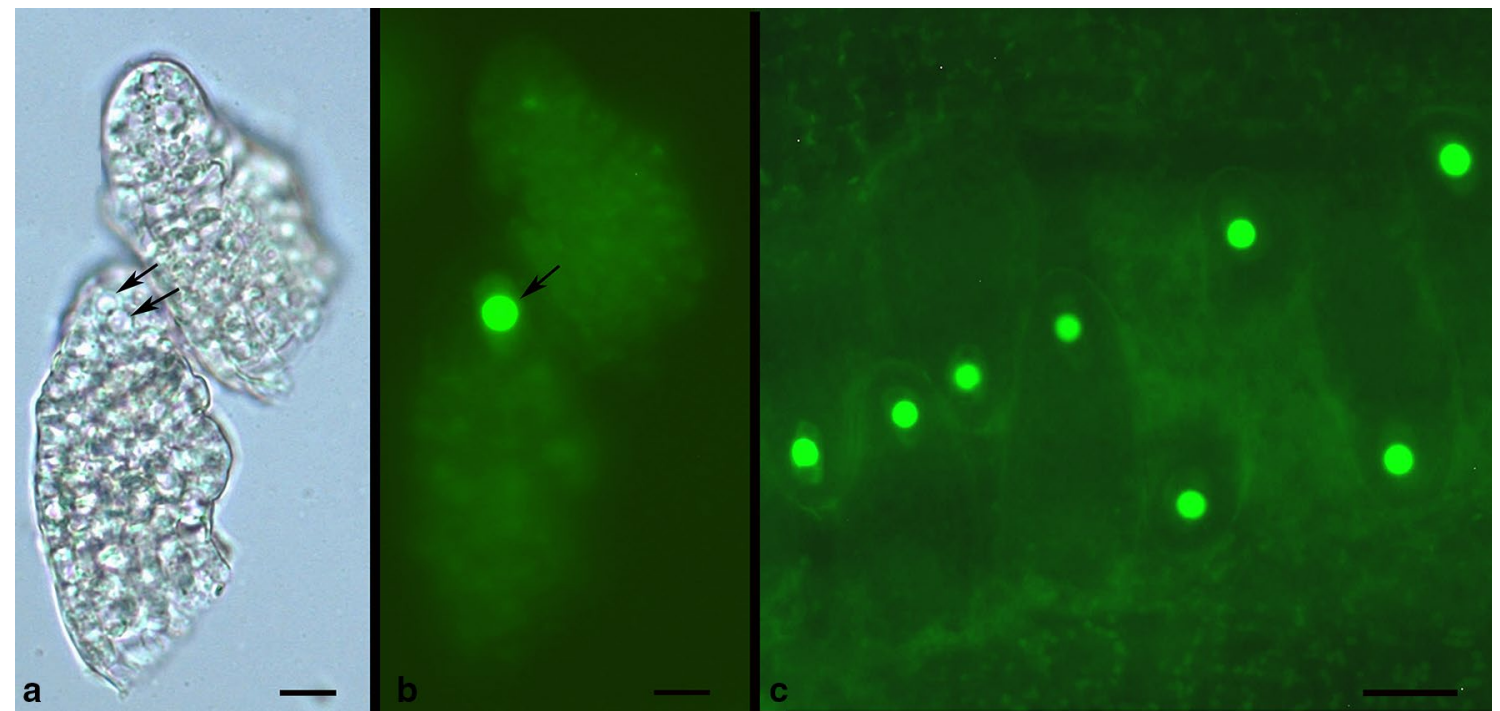

Fig. 9 Arabidopsis thaliana ovules of $p K N U: n l s Y F P$ line with the MMC YFP-labeled overexpressing AtGIDla under the control of p35ScaMV. a Ovules at the 2-III stage with two MMC-like cells (arrows) and $\mathbf{b}$ at the same moment using UV light showed signal of

comparable to what happens in plants of the $p K N U: n l s Y F P$ line, suggesting the possibility of identity other than with MMC to the MMC-like cells. It is important to note that the KNUCKLES promoter was not previously tested for identity of MMC-like cells.

Gallego-Giraldo et al. (2014) previously verified the differential expression of AtGIDIa, AtGIDIb and AtGIDIc in ovules, at anthesis, using GUS staining. Expression of AtGIDla was detected in all tissues of the ovules, with higher expression observed in the surrounding of the ES, while $A t G I D I b$ expression was observed mainly in the chalaza zone, at the base of the ES, and AtGIDIc was undetectable in the ovules. In our study, we detected AtGIDIa, AtGIDIb and AtGIDIc expression reported by GUS assays during the early stages of ovule development, before anthesis. AtGIDI $a$ and AtGIDIb were localized in the inner and outer integuments, not in MMCs and the surrounding nucellar cells. Expression signal of AtGIDIc was not detected in the ovules, before anthesis. Our results suggested that AtGIDI $a$ and AtGIDIb expression is associated with integument development in Arabidopsis. The association of the gibberellin and integument development was previously described (Gomez et al. 2016). In plants overexpressing AtGIDla, in comparison to the wild-type plants, an asynchrony between integument development and the stage of gametogenesis suggests that the ectopic expression of AtGIDla resulted in either a small delay in megasporogenesis or a faster growth of integuments in the transgenic plants. Consistent with the importance of gibberellin in the correct seed development to form seed-filled siliques, overexpressed fluorescence only in the MMC (arrow), not in the additional enlarged cells of the overexpressed plants. c Ovules finger-like (2-I) stage, showing yellow florescence in the MMC cells. $M M C$ megaspore mother cell; scale bars a-c $10 \mu \mathrm{m}$

AtGIDla plants were fertile without any sign of ovule or seed abortion and their siliques were seed-filled.

We demonstrated GID1 expression during megasporogenesis in ovules of sexual and apomictic $B$. brizantha, especially in the MMC. Specifically in ovules of the apomictic plants, BbrizGIDl is also expressed in the nucellar cells, previously to aposporous initial cell differentiation. In Arabidopsis, overexpression of AtGIDla triggered the differentiation of MMC-like cells in the nucellar region. These data suggest the involvement of GIDl in the early events of ovule and ES development.

Author contribution statement LGF, DMAD, ASTI, LC and VTCC designed the project; LGF, DMAD, ASTI, ACMMG, MAM and VTCC performed experimental procedures; LGF, DMAD, MAM, LC and VTCC, participated in the research, analyzed the data and wrote the paper. All authors read and approved the final manuscript.

Acknowledgements The authors acknowledge Dr. Perez-Amador who provided seeds of pAtGIDla:AtGIDla-GUS, pAtGIDIb: AtGID1b-GUS and pAtGID1c:AtGID1c-GUS transgenic lines and Dr. Tucker who supplied seeds of $p K N U: n l s Y F P$ line. We also thank the assistance of Simona Masiero, Lilian Florentino and Júlio Rodrigues in the laboratory; Roberto Togawa and Priscila Grynberg in the bioinformatics analysis; and Joseane Padilha in the statistical analyses. This work was supported by the National Council for Scientific and Technological Development-CNPq (449636/2014-3-VTCC) and the Brazilian Agricultural Research Corporation-Embrapa (02140101400.00VTCC). This work is part of Luciana Gomes Ferreira's PhD thesis from Pós-Graduação em Biologia Molecular, University of Brasilia-UnB, 
Brazil, with fellowship from Coordination for the Improvement of Higher Level Personnel-University of Brasília, CAPES-UnB, which also provided a 1-year fellowship at Università degli Studi di Milano, Italy. Foundation for Research Support of the Federal District-FAPDF provided financial assistance for LGF's participation in congresses.

\section{Compliance with ethical standards}

Conflict of interest The authors declare that they have no conflict of interest.

\section{References}

Altschul SF, Gish W, Miller W, Myers E, Lipman DJ (1990) Basic local alignment search tool. J Mol Biol 215:403-410

Altschul SF, Madden TL, Schaffer AA, Zhang J, Zhang Z, Miller W et al (1997) Gapped BLAST and PSI-BLAST: a new generation of protein database search programs. Nucleic Acid Res 25:3389-3402

Alves ER, Carneiro VTC, Dusi DMA (2007) In situ localization of three cDNA sequences associated with the later stages of aposporic embryo sac development of Brachiaria brizantha. Protoplasma 231:161-171

Araujo ACG, Mukhambetzhanov S, Pozzobon MT, Santana EF, Carneiro VTC (2000) Female gametophyte development in apomictic and sexual Brachiaria brizantha (Poaceae). Revue Cytol Biol Vég Le Bot 23:13-26

Araujo ACG, Falcão R, Carneiro VTC (2007) Seed abortion in the sexual counterpart of Brachiaria brizantha apomicts (Poaceae). Sex Plant Reprod 20:109-121

Bartrina I, Otto E, Strnad M, Werner T, Schmülling T (2011) Cytokinin regulates the activity of reproductive meristems, flower organ size, ovule formation, and thus seed yield in Arabidopsis thaliana. Plant Cell 23:69-80

Benfey PN, Ren L, Chua N (1990) Tissue-specific expression from CaMV 35S enhancer subdomains in early stages of plant development. EMBO J 9:1677-1684

Brambilla V, Kater M, Colombo L (2008) Ovule integument identity determination in Arabidopsis. Plant Signal Behav 3:246-247

Carneiro VTC, Dusi DMA, Ortiz JPA (2006) Apomixis: occurrence, applications and improvements. In: Silva JAT (ed) Floriculture, ornamental and plant biotechnology: advances and tropical issues, vol 01. Global Science Books, Ikenobe, pp 564-570

Chenna R, Sugawara H, Koike T, Lopez R, Gibson TJ, Higgins DG et al (2003) Multiple sequence alignment with the clustal series of programs. Nucleic Acids Res 31:3497-3500

Clough SJ, Bent AF (1998) Floral dip: a simplified method for agrobacterium-mediated transformation of Arabidopsis thaliana. Plant J 16:735-743

Cucinotta M, Colombo L, Roig-Villanova I (2014) Ovule development, a new model for lateral organ formation. Front Plant Sci $5: 1-12$

Davière J-M, Achard P (2013) Gibberellin signaling in plants. Development 140:1147-1151

Dellaporta SL, Wood J, Hicks BJ (1983) A plant DNA minpreparation: version II. Plant Mol Biol Rep 1:19-21

Dusi DMA, Willemse MTM (1999) Apomixis in Brachiaria decumbens Stapf.: gametophytic development and reproductive calendar. Acta Biol Cracov Ser Bot 41:151-162

Gallego-Giraldo C, Hu J, Urbez C, Gomez MD, Sun TP, Perez-Amador MA (2014) Role of the gibberellin receptors GID1 during fruit-set in Arabidopsis. Plant J 79:1020-1032
Gomez MD, Ventimilla D, Sacristan R, Perez-Amador MA (2016) Gibberellins regulate ovule integument development by interfering with the transcription factor ATS. Plant Physiol 172:2403-2415

Griffiths J, Murase K, Rieu I, Zentella R, Zhang Z-L, Powers SJ et al (2006) Genetic characterization and functional analysis of the GID1 gibberellin receptors in Arabidopsis. Plant Cell 18:3399-3414

Grossniklaus U, Schneitz K (1998) The molecular and genetic basis of ovule and megagametophyte development. Cell Dev Biol 9:227-238

Gupta R, Chakrabarty SK (2013) Gibberellic acid in plant: still a mystery unresolved. Plant Signal Behav 8:9

Hartweck LM, Olszewski NE (2006) Rice GIBBERELLIN INSENSITIVE DWARF1 is a gibberellin receptor that illuminates and raises questions about GA signaling. Plant Cell 18:278-282

Kooiker M, Airoldi CA, Losa A, Manzotti PS, Finzi L, Kater MM (2005) BASIC PENTACYSTEINE1, a GA binding protein that induces conformational changes in the regulatory region of the homeotic Arabidopsis gene SEEDSTICK. Plant Cell 17:722-729

Leblanc O, Armstead I, Pessino S, Ortiz JPA, Evans C, do Valle C et al (1997) Non-radioactive mRNA fingerprinting to visualise gene expression in mature ovaries of Brachiaria hybrids derived from B. brizantha, an apomictic tropical forage. Plant Sci 126:49-58

Liljegren SJ, Ditta GS, Eshed Y, Savidge B, Bowman JL, Yanofsky MF (2000) SHATTERPROOF MADS-box genes control seed dispersal in Arabidopsis. Nature 404:766-770

Nakajima M, Shimada A, Takashi Y, Kim YC, Park SH, Ueguchi-Tanaka $\mathrm{M}$ et al (2006) Identification and characterization of Arabidopsis gibberellin receptors. Plant J 46:880-889

Olmedo-Monfil V, Duran-Figueroa N, Arteaga-Vazquez M, DemesaArevalo E, Autran D, Grimanelli D et al (2010) Control of female gamete formation by a small RNA pathway in Arabidopsis. Nature 464:629-633

Osterlund T (2001) Structure-function relationships of hormone-sensitive lipase. Eur J Biochem 1907:1899-1907

R Core Team (2016) R: a language and environment for statistical computing. R Foundation for Statistical Computing, Vienna, Austria. http://www.R-project.org/

Rodrigues JCM, Cabral GB, Dusi DMA, Mello LV, Rigden DJ, Carneiro VTC (2003) Identification of differentially expressed cDNA sequences in ovaries of sexual and apomictic plants of Brachiaria brizantha. Plant Mol Biol 53:745-757

Rodríguez-Leal D, León-Martínez G, Abad-Vivero U, Vielle-Calzada J (2015) Natural variation in epigenetic pathways affects the specification of female gamete precursors in Arabidopsis. Plant Cell 27:1034-1045

Rozen S, Skaletsky H (2000) Primer3 on the WWW for general users and for biologist programmers. Methods Mol Biol 132:365-385

Sambrook J, Russell D (2001) Molecular cloning: a laboratory manual, 3rd edn. Cold Spring Harbor Laboratory Press, New York

Schneitz K, Hulskamp M, Pruitt RE (1995) Wild-type ovule development in Arabidopsis thaliana: a light microscope study of cleared whole-mount tissue. Plant J 7:731-749

Silveira ED, Alves-Ferreira M, Guimaraes LA, da Silva FR, Carneiro VTC (2009) Selection of reference genes for quantitative realtime PCR expression studies in the apomictic and sexual grass Brachiaria brizantha. BMC Plant Biol 9:84

Silveira ED, Guimaraes LA, Dusi DMA, da Silva FR, Martins NF, Costa MMC, Ferreira MA, Carnerio VTC (2012) Expressed sequence-tag analysis of ovaries of Brachiaria brizantha reveals genes associated with the early steps of embryo sac differentiation of apomictic plants. Plant Cell Rep 31:403-416

Sun T (2010) Gibberellin-GID1-DELLA: a pivotal regulatory module for plant growth and development. Plant Physiol 154:567-570

Suzuki H, Park S, Okubo K, Kitamura J, Ueguchi-Tanaka M, Iuchi S (2009) Differential expression and affinities of Arabidopsis 
gibberellin receptors can explain variation in phenotypes of multiple knock-out mutants. Plant J 60:48-55

Tucker MR, Okada T, Hu Y, Scholefield A, Taylor JM, Koltunow AMG (2012) Somatic small RNA pathways promote the mitotic events of megagametogenesis during female reproductive development in Arabidopsis. Development 1404:1399-1404

Ueguchi-Tanaka M, Ashikari M, Nakajima M, Itoh H, Katoh E, Kobayashi $\mathrm{M}$ et al (2005) GIBBERELLIN INSENSITIVE DWARF1 encodes a soluble receptor for gibberellin. Nature 437:693-698

Ueguchi-Tanaka M, Nakajima M, Motoyuki A, Matsuoka M (2007) Gibberellin receptor and its role in gibberellin signaling in plants. Annu Rev Plant Biol 58:183-198
Voegele A, Linkies A, Müller K, Leubner-Metzger G (2011) Members of the gibberellin receptor gene family GID1 (GIBBERELLIN INSENSITIVE DWARF1) play distinct roles during Lepidium sativum and Arabidopsis thaliana seed germination. J Exp Bot 62:5131-5147

Yang W-C, Shi D-Q, Chen Y-H (2010) Female gametophyte development in flowering plants. Annu Rev Plant Biol 61:89-108 